\title{
THE BIG PICTURE FROM RADIO WAVES TO GAMMA RAYS
}

\author{
C.D. IMPEY \\ Steward Observatory \\ University of Arizona, Tucson, AZ 85721, USA
}

\section{Introduction}

The conventional paradigm of active galactic nuclei (AGN) holds that they are powered by accretion onto a gravitational engine. In addition, the presence of Doopler-boosted radiation from jets and anisotropic obscuration can create large differences between the observed and intrinsic properties of AGN (Antonucci 1994). The "big picture"of extragalactic radio sources must include observations across the electromagnetic spectrum. Relativistic processes produce radiation with a very wide bandwidth. In addition, much of the energy from AGN is reprocessed, and the opacity is a function of wavelength. Finally, it turns out that only a small fraction of the bolometric luminosity of most AGN is emitted in the traditional radio and optical bands.

\section{Quasars and Blazars}

Samples of strong radio sources selected at high frequencies have the best complementary information at other wavelengths. For example, the roughly 300 quasars in the Kühr (1981) catalog now have virtually complete redshift information. In addition, about $70 \%$ of the quasars have optical polarimetry. After controlling for luminosity, strong optical and far infrared emission is correlated with the compactness of the radio source. High optical polarization, a clear indicator of nonthermal emission, is strongly correlated with radio compactness. Sources with these collective properties are called "blazars." It is also possible to distinguish highly polarized quasars, where normal quasar broad lines are superimposed on a polarized continuum, and BL Lac objects, where the lines are weak or absent. Among quasars stronger than $1 \mathrm{Jy}$ at $5 \mathrm{GHz}$, the proportion of highly polarized objects falls from 
$60 \%$ at $z=0.3$ to just under $20 \%$ at $z=2.7$. The BL Lac fraction in the 1 Jy catalog falls more steeply, from $30 \%$ at $z=0.3$ to under $10 \%$ at $z=1.5$.

These observations can be explained by a model where the radio and optical emission is beamed with a similar Lorentz factor and geometry, presuming that the optical emission is a sum of power law synchrotron and thermal accretion disk components. The decline in the fraction of highly polarized objects with increasing redshift results from the larger fraction of the emission which is due to the hot thermal component in the optical passband. This simple model works well for a power law spectral index of $\alpha=-1.5$, where $S_{\nu} \propto \nu^{\alpha}$. As expected, low polarization quasars are brighter than high polarization quasars, and radio-to-optical spectral index steepens with redshift, due to the dependence of the beamed power on local spectral index. BL Lac objects form a distinct class. No thermal components are seen, even at minimum light, so the rapid decline in redshift must be intrinsic to the population. Compact radio structure is an excellent predictor of nonthermal optical/infrared properties.

\section{Strong Gamma Ray Emission}

Gamma ray emission has been detected from a significant number of AGN (Montigny et al. 1995); over half of the sources at high galactic latitude in the second EGRET catalog are associated with strong radio sources, typically blazars (Thompson et al. 1995). About 35 of these firm identifications are in the $1 \mathrm{Jy}$ sample. When the spectral energy distributions are normalized to the radio band, it is found that the gamma ray flux is much more closely correlated with the radio flux than with the optical or ultraviolet flux. This may favor models where gamma ray photons are multiply upscattered from much lower energies, rather than from optical/UV seed photons. The correlation between radio flux, $S_{r}$, and (non-simultaneous) gamma ray flux, $S_{\gamma}$, is weak but significant. The ratio $S_{\gamma} / S_{r}$ is influenced by the large number of upper limits, but the $95 \%$ confidence range is $0.0038<S_{\gamma} / S_{r}<0.018$. Integrating the source counts, and taking account of variability, the contribution of AGN to the $100 \mathrm{MeV}$ background is $46_{-23}^{+54} \%$. Given the radio weakness of the more ubiquitous optically selected quasar, they must contribute less than $5 \%$ of the gamma ray background.

This work was partially supported by NSF grant AST 93-20715.

\section{References}

Antonucci, R.R. 1994, Ann.Rev.Ast.Ap., 31, 473

Kühr, H. et al. 1981, Ast.Ap.Supp., 45, 367

Montigny, C. et al. 1995, Ap.J., 440, 525

Thompson, D.J. et al. 1995, Ap.J.Supp., in press 